\title{
Correction to: The robustness of titanium hydride potassium perchlorate (THPP) for long-term stability of pyrotechnic mechanical devices (PMDs)
}

\author{
Junwoo Lee*, Hyuntae Choi*, Seyoung Lim*, Gil Hwan Ahn**, Jong Gyu Paik***, \\ Byung Tae Ryu ${ }^{* * *}$, Yong Ha Kim ${ }^{* * * *}$, and Yong Sun Won ${ }^{* * * *,+}$ \\ *Department of Chemical Engineering, Pohang University of Science and Technology (POSTECH), \\ Pohang, Kyoungbuk 37673, Korea \\ **Defence R\&D Center, Hanwha Corporation, Daejeon 34068, Korea \\ ***Agency for Defense Development, Daejeon 34183, Korea \\ ****Department of Chemical Engineering, Pukyong National University, Busan 48513, Korea \\ (Received 13 November 2018 • accepted 14 January 2019)
}

The article The robustness of titanium hydride potassium perchlorate (THPP) for long-term stability of pyrotechnic mechanical devices (PMDs), written by Junwoo Lee*, Hyuntae Choi*, Seyoung Lim*, Gil Hwan Ahn**, Jong Gyu Paik***, Byung Tae Ryu***, Yong Ha Kim****, and Yong Sun Won ${ }^{* * * * \dagger,}$, was originally pub- lished on the publisher's internet portal (currently SPringerLink) on 08 February 2019 with misprinted DOI number, 10.1007/s11814018-0228-8, due to the technical error from converting manuscript file from Microsoft Word to PDF. The correct DOI number for the article is 10.1007/s11814-019-0316-9.

${ }^{\dagger}$ To whom correspondence should be addressed.

E-mail: yswon@pknu.ac.kr

Copyright by The Korean Institute of Chemical Engineers. 